$02.1 ; 06.5$

\title{
Изменение температуры Дебая при аморфизации однокомпонентного вещества
}

\author{
(C) М.Н. Магомедов
}

Институт проблем геотермии ДагНЦ РАН, Махачкала, Россия

E-mail: mahmag4@mail.ru

Поступило в Редакцию 24 июня 2019 г.

В окончательной редакции 24 июня 2019 г.

Принято к публикации 3 июля 2019 г.

На основе нелинейной зависимости первого координационного числа от коэффициента упаковки структуры $\left(k_{p}\right)$ предложен метод расчета температуры Дебая для аморфной структуры однокомпонентного вещества. Исходя из параметров парного потенциала Ми-Леннард-Джонса рассчитаны температуры Дебая для кристаллической и аморфной структур ряда чистых металлов, алмаза, $\mathrm{Si}, \mathrm{Ge}$. Получено хорошее согласие с оценками других авторов. Показано, что при $k_{p}=0.45556$ достигается минимум удельной свободной энергии Гельмгольца, т. е. эта упаковка является термодинамически устойчивой аморфной структурой.

Ключевые слова: аморфизация, температура Дебая, металлы, кремний, германий.

DOI: 10.21883/PJTF.2019.20.48391.17944

Известно, что при низкотемпературной конденсации или при скоростном охлаждении расплава (закалке) многие однокомпонентные (чистые) вещества (металлы, $\mathrm{Si}, \mathrm{Ge}$ и др.) переходят в аморфное состояние $[1,2]$. Свойства аморфного металла существенно отличаются от свойств его кристаллического состояния. Но структура аморфного состояния определена не вполне точно, что приводит к широкому интервалу значений как первого координационного числа $\left(k_{n}\right)$, так и коэффициента упаковки $\left(k_{p}\right)$, которые характеризуют аморфную структуру $[3,4]$.

Для определения аморфной структуры нами использована функция $k_{n}\left(k_{p}\right)$, полученная нами в [5] на основании изучения зависимости $k_{n}\left(k_{p}\right)$ для четырех кубических структур: ГЦК $\left(k_{n}=12 ; k_{p}=\pi \cdot 2^{1 / 2} / 6=0.7405\right)$, ОЦК $\left(k_{n}=8 ; k_{p}=\pi \cdot 3^{1 / 2} / 8=0.6802\right)$, простой кубической $\left(k_{n}=6 ; k_{p}=\pi / 6=0.5236\right)$ и алмазной $\left(k_{n}=4\right.$; $\left.k_{p}=\pi \cdot 3^{1 / 2} / 16=0.3401\right)$. Оказалось, что с коэффициентом достоверности $R_{d}=1$ зависимость $k_{n}\left(k_{p}\right)$ описывается полиномом третьей степени следующего вида:

$$
\begin{aligned}
k_{n}\left(k_{p}\right)= & -71.76782+467.78914 k_{p}-925.48451 k_{p}^{2} \\
& +603.01146 k_{p}^{3} .
\end{aligned}
$$

Как видно из зависимости (1), графически представленной на рисунке, график $a$, она имеет $N$-петлю, и при $5.858 \leq k_{n} \leq 6.278$ и $0.4 \leq k_{p} \leq 0.624$ одному значению $k_{n}$ соответствуют два или три значения $k_{p}$. Поэтому данная область в работе [5] была определена как область „случайной упаковки“. Это согласуется с оценками других авторов для координационного числа в „случайной упаковке“: $k_{n}=6-6.08$ [1,3]. Мы полагаем, что область, лежащая между экстремумами на зависимости $k_{n}\left(k_{p}\right)$, соответствует аморфной структуре чистого металла, т. е. $0.45556 \leq k_{p}($ amor $p h) \leq 0.56762$ и $5.855 \leq k_{n}($ amor $p h) \leq 6.2793$.
Для подтверждения этой гипотезы необходимо рассчитать свойства металла в аморфном состоянии. Для этого положим, что атомы в металле взаимодействуют посредством парного потенциала Ми-Леннард-Джонса следующего вида $[6$, Гл. 3]:

$$
\varphi(r)=\frac{D}{(b-a)}\left[a\left(\frac{r_{o}}{r}\right)^{b}-b\left(\frac{r_{o}}{r}\right)^{a}\right],
$$

где $D$ и $r_{o}-$ глубина и координата минимума потенциала, $b>a>1$ - параметры.

Тогда, используя приближение „взаимодействия только ближайших соседей“, температуру Дебая как функцию $k_{n}$ (первого координационного числа) и $c=\left(6 k_{p} v / \pi\right)^{1 / 3}$ (расстояния между центрами ближайших атомов) можно определить в виде $[6$, Гл. 2; 7]:

$$
\Theta\left(k_{n}, c\right)=A_{w}\left(k_{n}, c\right) \xi\left[-1+\left(1+\frac{8 D}{k_{\mathrm{B}} A_{w}\left(k_{n}, c\right) \xi^{2}}\right)^{1 / 2}\right] .
$$

Здесь $k_{\mathrm{B}}$ - постоянная Больцмана, функция $A_{w}$ возникает из-за учета энергии „нулевых колебаний“ атомов в кристалле, $v=V / N-$ удельный объем, $V$ и $N-$ объем и число атомов в кристалле,

$$
\begin{gathered}
A_{w}\left(k_{n}, c\right)=K_{R} \frac{5 k_{n} a b(b+1)}{144(b-a)}\left(\frac{r_{o}}{c}\right)^{b+2}, \\
K_{R}=\frac{\hbar^{2}}{k_{\mathrm{B}} r_{o}^{2} m}, \quad \xi=\frac{9}{k_{\mathrm{n}}},
\end{gathered}
$$

где $m$ - масса атома, $\hbar$ - постоянная Планка.

Используя для колебательного спектра кристалла модель Эйнштейна, для удельной (на атом) свободной энергии Гельмгольца можно принять выражение 

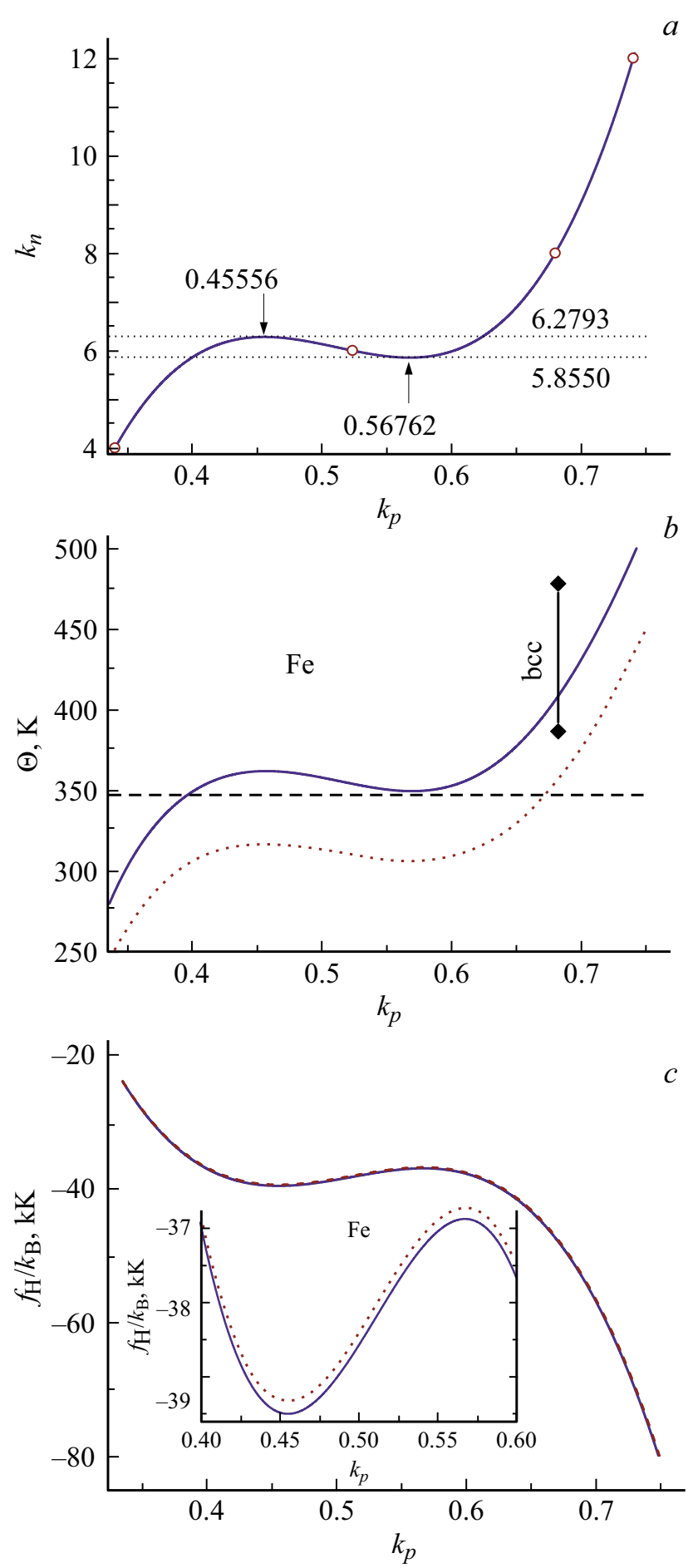

Зависимости от коэффициента упаковки первого координационного числа $(a)$, температуры Дебая $(b)$ и удельной свободной энергии Гельмгольца $(c)$.

вида $[6,8]$ :

$$
\begin{aligned}
& f_{\mathrm{H}}\left(k_{n}, c, T\right)=\left(\frac{k_{n}}{2}\right) D U(R)+3 k_{\mathrm{B}} \Theta_{\mathrm{E}}\left(k_{n}, c\right) \\
& \times\left\{\left(\frac{1}{2}\right)+\left(\frac{T}{\Theta_{\mathrm{E}}\left(k_{n}, c\right)}\right) \ln \left[1-\exp \left(-\frac{\Theta_{\mathrm{E}}\left(k_{n}, c\right)}{T}\right)\right]\right\},
\end{aligned}
$$

где $\Theta_{\mathrm{E}}-$ температура Эйнштейна, которая связана с температурой Дебая следующим соотношением [6]: $\Theta=(4 / 3) \Theta_{\mathrm{E}} ; R=r_{o} / c-$ относительная линейная плотность кристалла; функция потенциальной энергии в соответствии с (2) равна $U(R)=\left(a R^{b}-b R^{a}\right) /(b-a)$.

Путем подстановки функции (1) в (3), (4) были рассчитаны зависимости температуры Дебая $\Theta$ (см. рисунок, $b$ ) и удельной свободной энергии Гельмгольца $f_{\text {H }}$ (см. рисунок, $c$ ) от $k_{p}$ для $\mathrm{Fe}$. Параметры парного межатомного потенциала Ми-Леннард-Джонса для $\mathrm{Fe}$ были определены и протестированы в [9]. Расчеты были выполнены при температуре $300 \mathrm{~K}$ для двух значений расстояния между ближайшими атомами: $c / r_{o}=1$ (сплошная линия) и $c / r_{o}=1.0265$ (пунктир на графиках $b$ и $c$ рисунка).

Здесь $r_{o}(\mathrm{OЦК-Fe})=2.4775 \cdot 10^{-10} \mathrm{~m}-$ координата минимума потенциала из работы [9].

Для аморфного $\mathrm{Fe}$ в этих условиях получено:

$$
\begin{aligned}
& 348.84 \leq \Theta_{a m} \leq 361.32 \mathrm{~K} \text { при } c / r_{o}=1 ; \\
& 305.24 \leq \Theta_{a m} \leq 316.16 \mathrm{~K} \text { при } c / r_{o}=1.0265 .
\end{aligned}
$$

Это хорошо согласуется с оценками температуры Дебая для аморфного Fe из работы [2]: $\Theta_{\text {amor ph }}=346.2 \mathrm{~K}$ (горизонтальная прямая на рисунке, $b$ ). Для ОЦК-Fе температура Дебая лежит в интервале $386 \leq \Theta_{\mathrm{bcc}} \leq 478 \mathrm{~K}$ (показано вертикальной линией на графике, $b$ ).

Как видно из рисунка, график $c$ функция $f_{\mathrm{H}}$ (которая при нулевом давлении $P=0$ совпадает с удельным термодинамическим потенциалом Гиббса) образует $S$ петлю и имеет минимум при $k_{p}=0.45556$ и $k_{n}=6.2793$. Это указывает на то, что такая упаковка атомов является термодинамически устойчивой аморфной структурой.

При использовании параметров потенциала (2) из работ [6,10-13] с помощью формулы (3) были рассчитаны температуры Дебая при $R=r_{o} / c=1$ для кристаллического $\Theta_{c r}(R=1)$ и аморфного $\Theta_{a m}(R=1)$ состояний ряда чистых металлов, которые указаны в таблице. В двух правых столбцах показаны значения температуры Дебая для кристаллического $\Theta_{\text {crystal }}$ и аморфного $\Theta_{\text {amorph }}$ состояний, полученные для этих металлов в работах $[2,14]$.

Для „неквантовых“ кристаллов, у которых энергия „нулевых колебаний“ много меньше глубины межатомного потенциала (2), т. е. при $D /\left(k_{\mathrm{B}} A_{w} \xi^{2}\right) \gg 1 / 8$, выражение (3) можно упростить к виду $\Theta \cong\left(8 D A_{w} / k_{\mathrm{B}}\right)^{1 / 2}$. Тогда легко получить соотношение

$$
\Theta^{*}=\frac{\Theta_{a m}}{\Theta_{c r}} \cong\left(\frac{D_{a m} k_{n a m}}{D_{c r} k_{n c r}}\right)^{1 / 2}\left(\frac{c_{a m}}{c_{c r}}\right)^{-(b+2) / 2}
$$

Полагая, что при аморфизации моноатомного металла или диэлектрика изменение глубины парного межатомного потенциала и расстояния между центрами ближайших атомов незначительно, т.е. $D_{a m} \cong D_{c r}, c_{a m} \cong c_{c r}$, 
Параметры парного межатомного потенциала (2), рассчитанные при $R=r_{o} / c=1$ значения температур Дебая для кристаллического $\Theta_{c r}(R=1)$ и аморфного $\Theta_{a m}(R=1)$ состояний металлов с ГЦК- $\left(k_{n}=12\right)$, ГПУ- $\left(k_{n}=12^{*}\right)$ и ОЦК- $\left(k_{n}=8\right)$ структурами

\begin{tabular}{|c|c|c|c|c|c|c|c|c|c|c|}
\hline Металл & $k_{n}$ & $m$, a.m.u. & $r_{o}, 10^{-10} \mathrm{~m}$ & $D / k_{\mathrm{B}}, \mathrm{K}$ & $b$ & $a$ & $\Theta_{c r}(R=1), \mathrm{K}$ & $\Theta_{a m}(R=1), \mathrm{K}$ & $\Theta_{\text {crystal }}, \mathrm{K}$ & $\Theta_{\text {amorph }}, \mathrm{K}$ \\
\hline $\mathrm{Cu}$ & 12 & $\begin{array}{l}63.546 \\
63 \\
65\end{array}$ & $\begin{array}{c}2.5487 \\
-/ /- \\
-/ /-\end{array}$ & $\begin{array}{c}6841.30 \\
-/ /- \\
-/ /-\end{array}$ & $\begin{array}{l}8.37 \\
-/ /- \\
-/ /-\end{array}$ & $\begin{array}{l}3.03 \\
-/ /-[6] \\
-/ /-\end{array}$ & $\begin{array}{l}343.7 \\
345.2 \\
339.9\end{array}$ & $\begin{array}{l}239.6-248.2 \\
240.6-249.2 \\
236.9-245.4\end{array}$ & $\begin{array}{l}343 \\
351 \\
320\end{array}$ & $\begin{array}{l}268.8[2] \\
295.0[14] \\
268.0[14]\end{array}$ \\
\hline $\mathrm{Ag}$ & 12 & 107.868 & 2.8760 & 5737.19 & 10.35 & $3.08[6]$ & 226.6 & $158.1-163.7$ & $\begin{array}{l}224 \\
221\end{array}$ & $\begin{array}{l}154.5[2] \\
185.6[14]\end{array}$ \\
\hline $\mathrm{Au}$ & 12 & 196.967 & $\begin{array}{l}2.8751 \\
-/ /- \\
-/ /-\end{array}$ & $\begin{array}{c}7411.50 \\
-/ /- \\
-/ /-\end{array}$ & $\begin{array}{l}15.56 \\
16.34 \\
12.82\end{array}$ & $\begin{array}{l}1.96[6] \\
1.89[6] \\
2.93[10]\end{array}$ & $\begin{array}{l}165.0 \\
164.8 \\
196.1\end{array}$ & $\begin{array}{l}115.1-119.2 \\
115.0-119.1 \\
136.8-141.7\end{array}$ & 165 & $115.2[2]$ \\
\hline $\mathrm{Pb}$ & 12 & 207.20 & 3.4770 & 3678.47 & 14.50 & $2.52[6]$ & 105.7 & $73.7-76.4$ & 105 & $62.8[2]$ \\
\hline $\mathrm{Al}$ & 12 & 26.982 & 2.8541 & 6631.02 & 10.92 & $2.49[6]$ & 430.4 & $299.9-310.6$ & $\begin{array}{l}428 \\
400\end{array}$ & $\begin{array}{l}276.6[2] \\
336.0[14]\end{array}$ \\
\hline$\alpha-Y$ & $12^{*}$ & 88.906 & 3.5846 & 8546.35 & 9.61 & $3.55[6]$ & 268.0 & $187.0-193.7$ & 214 & $180.0[12]$ \\
\hline $\mathrm{Nb}$ & 8 & 92.9064 & 2.8648 & 21706.44 & 7.50 & $1.72[6]$ & 240.9 & $206.0-213.4$ & 275 & $185.6[2]$ \\
\hline $\mathrm{Cr}$ & 8 & 51.996 & $\begin{array}{l}2.4950 \\
-/ /-\end{array}$ & $\begin{array}{c}12117.91 \\
-/ /-\end{array}$ & $\begin{array}{l}7.12 \\
9.54\end{array}$ & $\begin{array}{l}4.30[6] \\
4.45[6]\end{array}$ & $\begin{array}{l}592.4 \\
591.5\end{array}$ & $\begin{array}{l}506.2-524.3 \\
505.5-523.6\end{array}$ & 630 & $416.3[2]$ \\
\hline Mo & 8 & 95.94 & $\begin{array}{l}2.7200 \\
-/ /-\end{array}$ & $\begin{array}{l}19776.91 \\
48950.00\end{array}$ & $\begin{array}{l}7.68 \\
6.82\end{array}$ & $\begin{array}{l}1.93[6] \\
1.87[11]\end{array}$ & $\begin{array}{l}258.8 \\
386.6\end{array}$ & $\begin{array}{l}221.3-229.2 \\
330.7-342.4\end{array}$ & $\begin{array}{l}450 \\
377\end{array}$ & $\begin{array}{l}319.6[2] \\
317.0[14]\end{array}$ \\
\hline $\mathrm{W}$ & 8 & 183.85 & $\begin{array}{l}2.7365 \\
-/ /-\end{array}$ & $\begin{array}{c}25608.93 \\
-/ /-\end{array}$ & $\begin{array}{l}8.58 \\
6.28\end{array}$ & $\begin{array}{l}4.06[6] \\
3.97[12]\end{array}$ & $\begin{array}{l}383.9 \\
396.1\end{array}$ & $\begin{array}{l}328.3-340.1 \\
338.7-350.8\end{array}$ & 400 & $255.5[2]$ \\
\hline$\alpha$-Fe & 8 & 55.847 & 2.4775 & 2576.700 & 8.26 & $2.95[9]$ & 408.1 & $348.8-361.3$ & 470 & $346.2[2]$ \\
\hline$\gamma$-Fe & 12 & $-/ /-$ & 2.5445 & 8384.467 & $-/ /-$ & $2.12[13]$ & 313.6 & $218.7-226.5$ & & \\
\hline
\end{tabular}

из (5) получим

$$
\begin{aligned}
& \Theta^{*} \cong\left(\frac{k_{n a m}}{k_{n c r}}\right)^{1 / 2}= \\
& \left\{\begin{array}{l}
{[(5.855-6.2793) / 12]^{1 / 2}=0.6985-0.7234 \text { для } k_{n c r}=12,} \\
{[(5.855-6.2793) / 8]^{1 / 2}=0.8555-0.886 \text { для } k_{n c r}=8 .}
\end{array}\right.
\end{aligned}
$$

Эти значения (6) согласуются с оценками величины

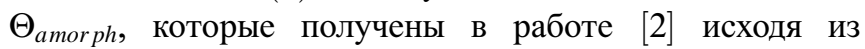
значений скорости звука и в работе [14] на основании измерения коэффициента теплового расширения аморфных металлов.

Что касается таких элементарных полупроводников, как алмаз, Si и Ge, то для них, как это было показано в [15], возможно два значения глубины парного межатомного потенциала (2): $D_{b}$ - глубина потенциала, который используют для расчета свойств при упругой (обратимой) деформации ковалентного кристалла, $D_{s}$ - глубина потенциала для расчета свойств кристалла при его пластической (необратимой) деформации. Полагая, как и авторы [14], что при аморфизации происходит необратимая деформация структуры, для $\mathrm{Si}$ $\left(D_{b}=5.54 \mathrm{eV}, D_{s}=2.32 \mathrm{eV}, D_{b} / D_{s}=2.388[6,15]\right)$ и $\mathrm{Ge}$ $\left(D_{b}=4.03 \mathrm{eV}, D_{s}=1.94 \mathrm{eV}, D_{b} / D_{s}=2.077[6,15]\right)$ из формулы (5) при $c_{a m} \cong c_{c r}$ можно получить

$$
\begin{aligned}
\Theta^{*} & \cong\left(\frac{(5.855-6.2793) D_{a m}}{4 D_{b}}\right)^{1 / 2}=(1.21-1.253) \\
& \times\left(\frac{D_{s}}{D_{b}}\right)^{1 / 2}=\left\{\begin{array}{l}
0.783-0.8107 \text { для } \mathrm{Si}, \\
0.8395-0.869 \text { для } \mathrm{Ge} .
\end{array}\right.
\end{aligned}
$$

Это соотношение хорошо согласуется с оценками других авторов:

$$
\begin{gathered}
\Theta^{*}(\mathrm{Si})=441 / 645=0.694[2], \quad 0.82[16], \\
460 / 554.2-557 / 645=0.83-0.864[17], \\
487 / 625=0.779[18], \\
\Theta^{*}(\mathrm{Ge})=244.6 / 374=0.654[2], \\
315 / 374=0.842[14,19], \quad 0.84[16] .
\end{gathered}
$$

Для кристаллической фазы $\mathrm{Si}$ и $\mathrm{Ge}$ экспериментально получено $\Theta_{c r}(\mathrm{Si})=645 \pm 5 \mathrm{~K}, \Theta_{c r}(\mathrm{Ge})=374 \pm 2 \mathrm{~K}[20]$. Тогда из (7) следует $\Theta_{a m}(\mathrm{Si})=505-523 \mathrm{~K}$,

$\Theta_{a m}(\mathrm{Ge})=314-325 \mathrm{~K}$, что также хорошо согласуется с оценками из работ [2,14,16-19].

Используя (7), можно получить оценку $\Theta^{*}$ и для алмаза $\left(D_{b}=8.43 \mathrm{eV}, D_{s}=3.68 \mathrm{eV}, D_{b} / D_{s}=2.2908[6]\right)$ : $\Theta^{*}($ C-dia $)=0.7995-0.828$. Аморфный алмаз был экспериментально получен при $P>45 \mathrm{GPa}$, но до сих пор свойства его изучены мало [21]. 


\section{Выводы}

Предложен метод расчета температуры Дебая для аморфных моноатомных веществ, основанный на зависимости первого координационного числа от коэффициента упаковки структуры. Показано, что при аморфизации элементарного металла, диэлектрика или полупроводника температура Дебая уменьшается. Расчеты показали хорошее согласие с оценками других авторов $[2,14,16-19]$.

Показано, что функция удельной свободной энергии Гельмгольца имеет минимум при $k_{p}=0.45556$, т.е. такая упаковка является термодинамически устойчивой аморфной структурой. Это значение $k_{p}$ достаточно близко к оценке, полученной при моделировании системы жестких сфер методом Монте-Карло в [22]: жидкость и кристалл сосуществуют между точкой замерзания $\left(k_{p f}=0.494 \pm 0.002\right)$ и точкой плавления $\left(k_{p s}=0.545 \pm 0.002\right)$.

Поскольку при аморфизации металла величина $\Theta^{*}$ из (5) меньше единицы, по причинам, изложенным в [23], это должно увеличить $T_{c}$ - температуру перехода в сверхпроводящее состояние, что и наблюдается в экспериментах $[14,23]$.

На основании RP-модели нанокристалла, представленной в $[6,10,12]$, можно утверждать, что при данных $P-T$ условиях наночастица с аморфной структурой имеет более заметную размерную зависимость термодинамических свойств, чем изомерно-изоморфный (т.е. при одинаковом числе атомов и форме поверхности) ей нанокристалл.

\section{Благодарности}

Автор выражает благодарность Э.Н. Ахмедову, С.П. Крамынину, Н.Ш. Газановой и 3.М. Сурхаевой за плодотворные дискуссии и помощь в работе.

\section{Финансирование работы}

Работа выполнена при финансовой поддержке Российского фонда фундаментальных исследований в рамках научного проекта № 18-29-11013_мк и программы Президиума РАН (программа № I.13).

\section{Список литературы}

[1] Белащенко Д.К. Компьютерное моделирование жидких и аморфных веществ. М.: МИСИС, 2005. 408 с.

[2] Singh R.N., Ali I. // Int. J. Appl. Phys. Math. 2013. V. 3. N 4. P. 275-279. DOI: 10.7763/IJAPM.2013.V3.220

[3] Melnikov G., Emelyanov S., Ignatenko N., Manzhos O. // Key Eng. Mater. 2018. V. 781. P. 137-142.

DOI: $10.4028 /$ www.scientific.net/KEM.781.137

[4] Baule A., Morone F., Herrmann H.J., Makse H.A. // Rev. Mod. Phys. 2018. V. 90. N 1. P. 015006 (1-56).

DOI: $10.1103 /$ RevModPhys.90.015006
[5] Магомедов М.Н. // ЖСХ. 2008. Т. 49. В. 1. С. 164-167. DOI: $10.1007 / \mathrm{s} 10947-008-0021-8$

[6] Магомедов М.Н. Изучение межатомного взаимодействия, образования вакансий и самодиффузии в кристаллах. М.: Физматлит, 2010. 544 с.

[7] Магомедов М.Н. // ЖТФ. 2013. Т. 83. В. 9. С. 56-62. DOI: $10.1134 / \mathrm{S} 106378421309020 \mathrm{X}$

[8] Жирибалько Л. Статистическая физика твердого тела. М.: Мир, 1975. $383 \mathrm{c}$.

[9] Магомедов М.Н. // ЖТФ. 2015. Т. 85. В. 11. С. 48-54. DOI: $10.1134 / \mathrm{S} 1063784215110195$

[10] Ахмедов Э.Н. Зависимость свойств от размера и формы нанокристалла золота в изобарических условиях // Физико-химические аспекты изучения кластеров, наноструктур и наноматериалов. Тверь: Изд-во Твер. гос. ун-та, 2018. B. 10. C. $53-63$. DOI: $10.26456 /$ pcascnn $/ 2018.10 .053$

[11] Akhmedov E.N. // J. Phys. Chem. Solids. 2018. V. 121. P. 62 66. DOI: $10.1016 /$ j.jpcs.2018.05.011

[12] Gazanova N.Sh. // Appl. Solid State Chem. 2018. V. 3 (4). P. 36-40. DOI: 10.18572/2619-0141-2018-3-4-36-40

[13] Магомедов М.Н. // ЖТФ. 2017. Т. 87. В. 4. С. 549-556. DOI: $10.1134 / \mathrm{S} 1063784217040156$

[14] Троицкий О.А., Дринков А.В., Зайцев С.В. // Вопр. атомной науки и техники. Сер. Физика радиационных повреждений и радиационное материаловедение. 2011. Т. 98. В. 4. C. $132-135$.

[15] Магомедов М.Н. // ФТТ. 2017. Т. 59. В. 6. С. 1065-1072. DOI: $10.1134 / \mathrm{S} 1063783417060142$

[16] Mertig M., Pompe G., Hegenbarth E. // Solid State Commun. 1984. V. 49. N 4. P. 369-372. DOI: $10.1016 / 0038-1098(84) 90589-1$

[17] Feldman J.L., Allen P.B., Bickham S.R. // Phys. Rev. B. 1999. V. 59. N 5. P. 3551-3559. DOI: $10.1103 /$ physrevb.59.3551

[18] Zink B.L., Pietri R., Hellman F. // Phys. Rev. Lett. 2006. V. 96. N 5. P. 055902 (1-4). DOI: 10.1103/PhysRevLett.96.055902

[19] King C.N., Phillips W.A., de Neufville J.P. // Phys. Rev. Lett. 1974. V. 32. N 10. P. 538-541. DOI: 10.1103/PhysRevLett.32.538

[20] Flubacher P., Leadbetter A.J., Morrison J.A. // Phil. Mag. 1959. V. 4. N 39. P. 273-294. DOI: $10.1080 / 14786435908233340$

[21] Zeng Z., Yang L., Zeng Q., Lou H., Sheng H., Wen J., Miller D.J., Meng Y., Yang W., Mao W.L., Mao H.K. // Nature Commun. 2017. V. 8. P. 322 (1-7). DOI: $10.1038 / \mathrm{s} 41467-017-00395-\mathrm{w}$

[22] Hoover W.G., Ree F.H. // J. Chem. Phys. 1968. V. 49. N 8. P. 3609-3617. DOI: $10.1063 / 1.1670641$

[23] Garland J.W., Bennemann K.H., Mueller F.M. // Phys. Rev. Lett. 1968. V. 21. N 18. P. 1315-1319. DOI: $10.1103 /$ physrevlett.21.1315 